\title{
Impact of Prefabricated Versus 3-D Insoles on Pain and Quality of Life in Patients with Plantar Fasciitis
}

\author{
Rohina Kumari ${ }^{1}$, Surbhi ${ }^{2}$ \\ ${ }^{1}$ Lecturer, Department of Prosthetics and Orthotics, Ishwar Institute of Prosthetics and Orthotics, Anna Nagar, \\ Chennai, Tamil Nadu, India \\ ${ }^{2}$ Department of Prosthetics and Orthotics, Indian Spinal Injuries Center - Institute of Rehabilitation Sciences, \\ Vasant Kunj, New Delhi, India \\ Corresponding Author: Rohina Kumari
}

DOI: https://doi.org/10.52403/ijhsr.20220221

\begin{abstract}
Background: Plantar fasciitis is a condition in which the plantar fascia becomes inflamed. It causes discomfort and stiffness in the heel and medial arch of the foot, which can interfere with daily activities. Plantar fasciitis is commonly treated with insoles. Patient satisfaction with insoles has been demonstrated to improve symptoms in studies. However, just a few studies have been conducted to determine the effectiveness of using $3 \mathrm{D}$ insoles to treat PF.

Aims and Objective: To assess the effectiveness of prefabricated and 3-D insoles on pain and quality of life in patients with plantar fasciitis.

Methodology: Twenty subjects with plantar fasciitis were studied. All subjects had previously been prescribed and were using insoles to prevent the extent of pain. The impact of the insoles on pain was assessed by using VAS (visual analog scale) and quality of life was assessed by using American chronic pain association.

Result: Higher pain scores and reduced quality of life were recorded with prefabricated insoles when compared to customized $3 \mathrm{D}$ insoles.

Conclusion: This study demonstrates the efficacy of customized 3D printing insoles for reducing pain, improving quality of life and comfort in individuals with plantar fasciitis. In comparison to prefabricated insoles, customized 3D insoles are effective in the treatment of plantar fasciitis.
\end{abstract}

Keywords: plantar fasciitis, pain, quality of life, prefabricated insoles, 3D insoles

\section{BACKGROUND}

Plantar fasciitis is a common orthopaedic condition that causes pain in the plantar heel and makes weight bearing difficult. Chronic plantar heel pain affects over a million people in the United States each year. The plantar fascia is responsible for supporting the foot arch during walking. Microtrauma and plantar heel discomfort can be caused by excessive or recurrent traction of the fascia. Plantar fasciitis can be caused by pes planus or severe subtalar pronation, which overstretches the fascia. $^{1,2,3}$
Plantar fasciitis, also known as plantar heel pain (PHP), jogger's heel, tennis heel, and policeman's heel, is a painful syndrome that affects the calcaneus (heel bone). ${ }^{4}$

The most common symptoms are pain and stiffness, which appear gradually, especially during the first steps when getting out of bed in the morning. The severity of the symptoms varies and may improve after a few steps or within a few hours, but they frequently worsen as the day progresses. ${ }^{5}$

It is one of the most common chronic foot complaints that can cause dysfunction. Approximately $10 \%$ of the 
Rohina Kumari et.al. Impact of prefabricated versus 3-D insoles on pain and quality of life in patients with plantar fasciitis.

population suffers from heel pain at some point in their lives. Conservative management, such as stretching exercises, night splints, orthotics, insoles, extracorporeal shockwave therapy, and local injection, improves approximately $90 \%$ of patients in a year. Plantar fasciitis can be treated with inexpensive foot insoles. It can help people with plantar fasciitis who have a profession that requires a lot of walking or standing and don't have enough time to do stretching exercises or visit a doctor. Many different types of insoles have been introduced, but their effectiveness is questionable. According to some authors, prefabricated insoles are as effective as customized foot insoles. The customized foot insoles were found to be superior to prefabricated foot insoles in the majority of the study. ${ }^{6}$

Currently, new possibilities for insole manufacturing are emerging as a result of advancements in 3D technology. 3D printing is essentially a computer-aided design and manufacturing process (CADCAM). Fast construction and on-demand production are two advantages of 3D printing technology which meet the needs of individualized medical treatment. ${ }^{7}$ There is little scientific evidence to support the use of 3D insoles to treat PF. The aim of our present study was to assess the effect prefabricated and 3D insoles on pain, quality of life, and satisfaction in patients with PF.

\section{METHODOLOGY \\ Selection of Subjects}

This preliminary study was conducted in New Delhi, India. A Convenience sample of 20 plantar fasciitis patients who voluntarily participated in the study (descriptive information) given in Table 1. Out of 20 patients that were selected according to the inclusion criteria 13 were female and 7 were female with mean age of $43.15 \pm 9.41$ years, mean height of $167.72 \pm 7.50 \mathrm{~cm}$ and mean weight of $70.27 \pm 9.50 \mathrm{~kg}$.
Table 1. Descriptive Data of Mean Age, Height, Weight and BMI

\begin{tabular}{|l|l|l|}
\hline Subjects' characteristics & N & Mean \pm Std. Deviation \\
\hline Age & 20 & $43.15 \pm 9.4$ \\
\hline Height & 20 & $167.72 \pm 7.5$ \\
\hline Weight & 20 & $70.27 \pm 9.5$ \\
\hline BMI & 20 & $24.87 \pm 1.79$ \\
\hline \multicolumn{2}{|l}{} \\
*Significant at 0.05 level
\end{tabular}

\section{Inclusion Criteria}

Males or females with a diagnosis of $\mathrm{PF}$, aged 30-60 years, and the diagnosis was made upon the finding of tenderness to pressure at the origin of the plantar fascia on the calcaneus's medial tubercle, as well as a complaint of heel pain greater than or equal to 3 on a $1-10$ visual analogue scale (VAS).

\section{Exclusion criteria}

Previous fracture or surgery of the foot, specific metabolic and connective tissue disorders associated with or contributing to the diagnosis of PF (e.g., rheumatoid arthritis, gout, and lupus), hind foot deformity, Achilles tendonitis and subtalar arthritis.

\section{Procedure}

The parameters used in the study were VAS (visual analog scale) for pain and American chronic pain association for quality of life. Two groups were formulated under prefabricated and 3-D insoles and subjects were grouped according to the insoles prescribed. Each group consisted of 10 patients. The VAS and American chronic pain association score were recorded after 4 weeks of the prescription of insoles from each patient of the group.

\section{Statistical Technique}

The mean score of the parameters for each group were used for statistical analysis. A paired sample t-test was used for the pair wise comparison. SPSS software, version 21 , was used to analyze the data. The significance level was set at $\mathrm{P}<0.05$.

\section{RESULT}

Analysis using the paired sample ttest showed that 3D insoles are better in reducing pain and improving quality of life of the patient. The comparison in the value 
of pain reduction showed a significant difference, with prefabricated the value was $6.1 \pm 0.99$ and for $3 \mathrm{D}$ insoles it was 4.3 \pm 0.82 (Table 2, graph 1). The comparison in the value of quality of life score showed a significant difference, with prefabricated the value obtained were $6.3 \pm 0.67$ and for $3 \mathrm{D}$ insoles 8.1 \pm 0.73 . (Table 3, graph 2).

Table 2. VAS score of 3D and prefabricated insoles

\begin{tabular}{|c|c|c|c|}
\hline & $($ Mean \pm S.D $)$ & t-value & p-value \\
\hline VAS 3D & $4.3 \pm 0.82$ & \multirow[t]{2}{*}{6.194} & \multirow[t]{2}{*}{0.000} \\
\hline VAS PREFAB & $6.1 \pm 0.99$ & & \\
\hline
\end{tabular}

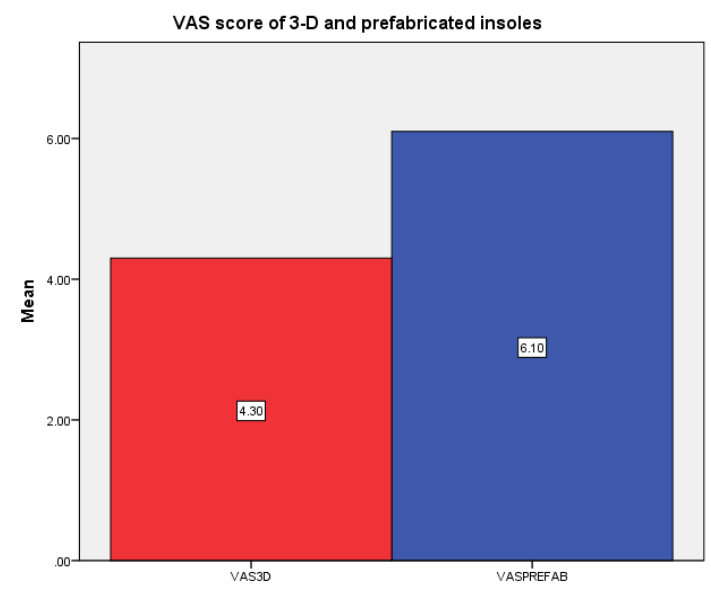

Graph 1

Table 3. QOL score of 3D and prefabricated insoles

\begin{tabular}{|l|l|l|l|}
\hline & $($ Mean \pm S.D) & t-value & p-value \\
\hline QOL 3D & $8.1 \pm 0.73$ & 7.216 & 0.000 \\
\hline QOL PREFAB & $6.3 \pm 0.67$ & & \\
\hline \multicolumn{4}{|c|}{ Values are represented as mean $\pm S D$} \\
& $*$ Significance at 0.05 level $*$
\end{tabular}

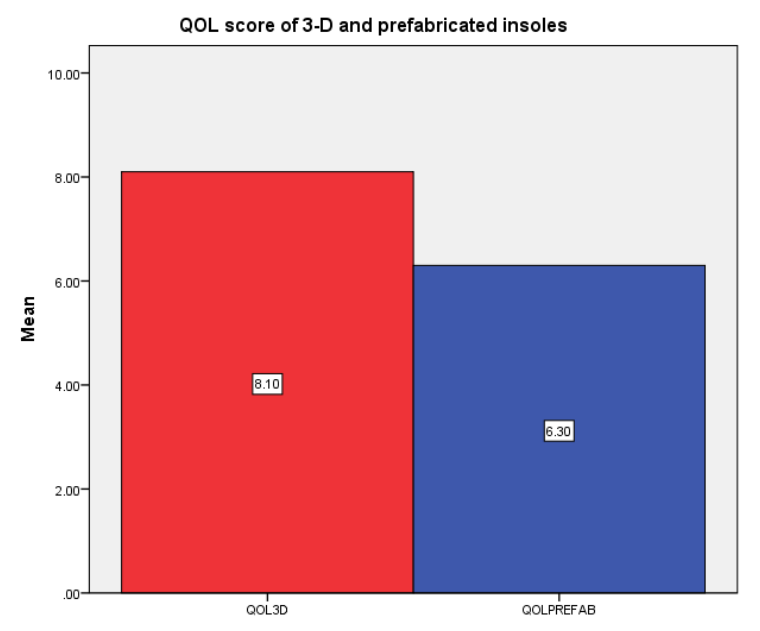

Graph 2

\section{DISCUSSION}

This study was targeted towards investigating the effect of two types of insoles on pain as well as quality of life. The conclusion extracted from the result shows that the 3-D insoles are better in respect to relief of pain and quality of life as well.

Mechanical treatment of plantar fasciitis appears to be beneficial in relieving symptoms of plantar fasciitis. None of the studies found a negative effect of mechanical treatment; they all found a positive or no effect. Insoles were the most researched treatment in the literature. Plantar fasciitis is treated with insoles of various shapes and materials. Insoles that cover the entire foot have a larger area of contact between the insole and the foot than heel cups. A larger contact area improves pressure distribution under the foot and, as a result, reduces peak pressures at the plantar fascia insertion. Furthermore, customized insoles support the longitudinal arch, which is associated with less plantar fascia strain. ${ }^{8-}$ 11

Foot orthosis have been shown in recent studies to reduce heel pain by relieving strain on the plantar fascia and reducing foot pronation and arch collapse. Heel pads can help to redistribute plantar pressure and alleviate the symptoms of plantar fasciitis. In order to alleviate plantar heel pain, customized insoles can reduce plantar pressure and transfer pressure from the hindfoot to the midfoot region. As a result, many different brands of customized insoles have been developed for patients suffering from plantar heel pain. ${ }^{11-15}$

Pain relief and comfort are obtained by customizing the insole, which provides localized pain relief. Support in an orthotic is critical for keeping the foot in an optimal position and preventing pronation, which contributes to fasciitis. The $3 \mathrm{D}$ insoles are specifically designed to prevent foot eversion and pronation based on the anatomy of the patient's foot. To summarize, the customized nature of the $3 \mathrm{D}$ insoles maximizes fit and compliance, while the softness increases comfort and 
effectiveness. It is proposed that the medial surface contours of the insoles must stabilize the apical bony structure of the foot arch in order to effectively support the longitudinal arch of the foot. ${ }^{13}$

The use of the foot orthosis is also influenced by its comfort. Uncomfortable orthosis have been shown to affect lower limb balance control and patient mood particularly in the elderly. 7,16,17

This study found that the customized 3D insoles provide significantly more comfort than the prefabricated insoles, and that it has a long-term effect, perhaps increasing treatment outcomes.

This study included the small sample size, Future studies with a larger sample size and more types of insoles would be preferable for a more precise view on insole selection.

\section{CONCLUSION}

According to the current findings, 3 $\mathrm{D}$ insoles can be used to reduce pain while walking in patients with PF. Overall, patients should be prescribed more customized $3 \mathrm{D}$ insoles because they better match the patient's foot anatomy than prefabricated insoles so they perform better.

\section{Acknowledgement: None}

\section{Conflict of Interest: None}

\section{Source of Funding: None}

\section{Ethical Approval: Approved}

\section{REFERENCES}

1. U CHHY, Hen CHENHC. Using an Optimization Approach to Design an Insole for Lowering Plantar Fascia Stress - A Finite Element Study. annals of biomedical engineering. 2008;36(8):1345-52.

2. Daniel L Riddle, Matthew Pulisic, Peter Pidcoe REJ. Risk factors for Plantar fasciitis: a matched case-control study. Journal of Bone and Joint Surgery - Series A. 2003;85(5):872-7.

3. Cole C, Seto C, Gazewood J. Plantar fasciitis: Evidence-based review of diagnosis and therapy. American Family Physician. 2005;72(11):2237-42.

4. Li L, Yang L, Yu F, Shi J, Zhu L, Yang X, et al. 3D printing individualized heel cup for improving the self-reported pain of plantar fasciitis. Journal of Translational Medicine. 2018;16(1):1-11.

5. Oliveira HAV, Jones A, Moreira E, Jennings F, Natour J. Effectiveness of total contact insoles in patients with plantar fasciitis. Journal of Rheumatology. 2015; 42(5):870-8.

6. Shim DW, Sung SY, Chung WY, Kang KY, Park SJ, Lee JW, et al. Superior pedal function recovery of newly designed three spike insole over total contact insole in refractory plantar fasciitis: A randomized, double-blinded, non-inferiority study. PLoS ONE. 2021;16:1-12.

7. Xu R, Wang Z, Ma T, Ren Z, Jin H. Effect of 3D printing individualized ankle-foot orthosis on plantar biomechanics and pain in patients with plantar fasciitis: A randomized controlled trial. clinical research. 2019;25:1392-400.

8. Schuitema D, Greve C, Postema K, Dekker R, Hijmans JM. Effectiveness of Mechanical Treatment for Plantar Fasciitis : A Systematic Review. journal of sport rehabilitation. 2020;29:657-74.

9. Chia JKK, Suresh S, Kuah A, Ong JLJ, Phua JMT, Seah AL. Comparative trial of the foot pressure patterns between corrective orthotics, formthotics, bone spur pads and flat insoles in patients with chronic plantar fasciitis. Annals of the Academy of Medicine Singapore. 2009;38(10):869-75.

10. Bonanno DR, Landorf KB, Menz HB. Pressure-relieving properties of various shoe inserts in older people with plantar heel pain. Gait and Posture. 2011;33(3):385-9.

11. Koglerco GF, Solomonidisc SE, Paul JP. Biomechanics of longitudinal arch support mechanisms in foot orthoses and their effect on plantar aponeurosis strain. Clinical Biomechanics. 1996;11(5):243-52.

12. Chen WP, Ju CW, Tang FT. Effects of total contact insoles on the plantar stress redistribution: A finite element analysis. Clinical Biomechanics. 2003;18(6):17-24.

13. Seligman DA, Dawson DR. Customized heel pads and soft orthotics to treat heel pain and plantar fasciitis. Archives of Physical Medicine and Rehabilitation. 2003;84(10): 1564-7. 
Rohina Kumari et.al. Impact of prefabricated versus 3-D insoles on pain and quality of life in patients with plantar fasciitis.

14. Kitaoka HB, Luo Z, Kura H, An K. Effect of Foot Orthoses on 3-Dimensional Kinematics of Flatfoot: A Cadaveric Study. arch phys med rehabil. 2002;83:876-9.

15. Kogler GF, Veer FB, Verhulst SJ, Solomonidis SE, Paul JP. The Effect of Heel Elevation on Strain Within the Plantar Aponeurosis: In Vitro Study. Foot \& Ankle International. 2001;22(5):433-9.

16. Cha YH, Lee KH, Ryu HJ, Joo IW, Seo A, Kim DH, et al. Ankle-foot orthosis made by 3D printing technique and automated design software. Applied Bionics and Biomechanics. 2017;1-7.
17. Zammit G V., Menz HB, Munteanu SE. Reliability of the TekScan MatScan ${ }^{\circledR}$ system for the measurement of plantar forces and pressures during barefoot level walking in healthy adults. Journal of Foot and Ankle Research. 2010;3(1):1-9.

How to cite this article: Kumari R, Surbhi. Impact of prefabricated versus 3-D insoles on pain and quality of life in patients with plantar fasciitis. Int J Health Sci Res. 2022; 12(2):153157. DOI: https://doi.org/10.52403/ijhsr. 20220221 